The Egyptian Journal of Hospital Medicine (July 2019) Vol. 76 (3), Page 3692-3700

\title{
Studying the Auditory Effect of Maternal Iron Deficiency Anemia on Neonates Using Otoacoustic Emissions (Oaes) \\ Islam Gamal Ali El-Diasty ${ }^{1}$, Hamed Mohamed El-Sharkawy ${ }^{1}$, Trandil Hassan Elmehalawi ${ }^{2}$ and Mona Khaled Abo Almaaty Omar ${ }^{3}$ \\ Department of Pediatric Medicine ${ }^{1}$, Department of Audio-Vestibular Medicine ${ }^{2}$, and Department of Obstetrics and Gynecology ${ }^{3}$, Faculty of Medicine, Tanta University, Egypt. \\ *Corresponding author: Islam Gamal Ali El-Diasty; Mobile: 01015214194; Email: islam_gamal@yahoo.com
}

\begin{abstract}
Background: The adverse effects of iron deficiency anemia on auditory nerve (AN) development have been showed a significant functional consequence on auditory brainstem response (ABR). Aim of the work was to study the effect of maternal iron deficiency anemia on auditory system of newborn infant using otoacoustic emissions (OAEs). Subjects and Methods: 60 full term neonates were divided into 2 groups: Group 1: 30 newborn infants as a control group with maternal blood $\mathrm{Hb}>10.5 \mathrm{~g} / \mathrm{dL}$. Group 2: study group: 30 newborn infants with maternal blood $\mathrm{Hb} \leq 10.5 \mathrm{~g} / \mathrm{dL}$ and diagnosed as maternal iron deficiency anemia (IDA). Results: In transient-evoked otoacoustic emissions (TEOAE) there was significant difference between the studied groups at frequency of $4000 \mathrm{~Hz}$ in both right and left ears. There was also significant difference between right and left ears as regard signal to noise ratio (SNR) and overall amplitude TEOAE at frequency of $2000 \mathrm{~Hz}$ in control group and at $4000 \mathrm{~Hz}$ in study group. There was significant difference in study group between right and left ears as regard signal to noise ratio (SNR) distortion-product otoacoustic emissions (DPOAE) at frequencies 1409 with right ears showing a higher mean SNR than left ears. Conclusion: The peripheral auditory function, as tested by TEOAEs test, is affected in high frequency region of the cochlea in low ferritin babies while DPOAEs test was less sensitive in these patients for assessment of their cochlear function.
\end{abstract}

Keywords: Maternal iron deficiency anemia, Neonates, Otoacoustic emissions.

\section{INTRODUCTION}

Anemia in pregnancy affects nearly half of all pregnant women worldwide and spreads in developing countries. The incidence of anemia in pregnancy in developing countries ranges from 53 to $61 \%$ for Africa, from 44 to $53 \%$ for South-East Asia, and from 17 to $31 \%$ for Europe and North America ${ }^{(1)}$. The prevalence of anemia among pregnant women in Egypt was $22.6 \%$ in 2016, according to the World Bank collection of development indicators ${ }^{(2)}$.

Previous reports indicate that hearing system is affected by iron deficiency, which may provide a pathological basis for deafness; on the other hand, iron deficiency may lead to functional and structural changes in the cochlea because some of the cochlear enzymes contain iron ${ }^{(3)}$.

Otoacoustic emissions (OAE) are low-intensity sounds emitted from the functioning outer hair cells of the cochlea. They can be measured even when the eighth nerve has been severed or blocked chemically ${ }^{(4)}$.

Aim of the work was to study the effect of maternal iron deficiency anemia on auditory system of newborn infant using otoacoustic emissions (OAEs).

\section{PATIENTS AND METHODS}

This case control study was carried out during the period from January 2017 to June 2018. Cases were selected from Obstetrics and Gynecology Department and Neonatology Unit, Tanta University Hospitals. It included 60 full term neonates who were divided into 2 groups: Group 1; control group: 30 newborn infants with maternal blood $\mathrm{Hb}>10.5 \mathrm{~g} / \mathrm{dL}$ as control infants and matched with age and sex for patient group. Group 2: study group: 30 newborn infants with maternal IDA with maternal blood $\mathrm{Hb} \leq 10.5 \mathrm{~g} / \mathrm{dL}^{(5)}$ and diagnosed as IDA according to $\mathrm{WHO}^{(6)}$.

Inclusion criteria: Full term neonates of iron deficiency anemia mothers without any other pregnancy associated diseases.

- The birth weight of babies was appropriate for gestational age.

- Maternal age between 19 and 40 years.

- Normal APGAR score of neonate at $1^{\text {st }}$ or $5^{\text {th }}$ minutes (>7/10).

- The babies with normal middle ear function test by high frequency tympanometry were assessed by (OAEs).

Exclusion criteria:

- Post term neonates (gestational age $\geq 42$ weeks).

- Neonates less than 37 weeks GA.

- Neonates small or large for gestational age.

- Low APGAR score (less than (7/10) at $1^{\text {st }}$ and $5^{\text {th }}$ minutes).

- Neonatal sepsis.

- Neonates with cephalohematoma or subgaleal bleeding.

- Neonates with respiratory distress. 
- IDM (infant of diabetic mother).

- Neonates with delayed $1^{\text {st }}$ cry or HIE (hypoxic ischemic encephalopathy).

- Neonates with congenital malformations, syndromes and chromosomal abnormalities.

- Neonates with infectious diseases as toxoplasmosis, rubella, cytomegalovirus, herpes, syphilis and human immuno-deficiency infections.

- Neonates with pathological jaundice or neonatal asphyxia or any birth trauma.

- Neonates with family history of hearing loss.

- Neonates died before completing laboratory investigations required for our research.

Ethical approval: Written Informed consent was obtained from the parents or guardians of the child. The study was approved by the Ethics Committee of Faculty of Medicine, Tanta University.

Collection of data: All infants and children were subjected to:

1. Complete History Taking: Including

a. Gestational age at delivery as calculated according to the last menstrual period and confirmed by ultrasound examination early in pregnancy.

b. Obstetric history (previous sibling death or admission to NICU).

c. Prenatal history (diabetes mellitus, maternal antibiotics, maternal urinary tract infections (UTI), drug intake, gestational diabetes, antenatal infections, preeclampsia or eclampsia and delayed cord clamping, maternal fever $>38^{\circ} \mathrm{c}$ ).

d. Family history of hearing loss, anemia, suspected immune deficiency diseases, metabolic disorders. Also we asked about history of a sibling with hearing or developmental problems.

e. Mode of delivery, resuscitation data and APGAR score at $1^{\text {st }}$ and $5^{\text {th }}$ minutes.

f. Natal history (PROM, maternal fever, trauma to the placental bed, feto-maternal hemorrhage, fever, offensive liquor, vaginal discharge, prolonged labor or obstructed labor, trauma to the newborn and asphyxia).

g. Postnatal history (aggressive resuscitation, respiratory distress, fever, jaundice, pallor, plethora, cyanosis, hypothermia, convulsions, delayed first respiratory distress, weight loss and stool and urine output).

h. Feeding history of breast feeding and any other supplements or drugs.

2. Thorough Clinical Examination: Including all body systems, vital data, general examination, chest, heart, abdomen, CNS, and complete local cardiac examination.

- Assessment of gestational age according to expanded new Ballard ${ }^{(7)}$.

- Measurement of weight, length and head circumference

- Vital signs including: temperature, respiratory rate and pulse.

- General appearance including: activity, skin color and presence of obvious congenital anomalies.

- Skin as regards: jaundice, pallor, plethora, cyanosis and mottling.

- Head as regards: anterior and posterior fontanels and evidence of increased intracranial pressure.

- Systemic examination as follows:

- Chest as regards: Symmetry, breath sounds and signs of respiratory distress.

- Heart as regards: heart rate, quality of heart sounds and presence of audible murmurs.

- Abdomen as regards: obvious defects, auscultation for bowel sounds.

- Genitalia for presence of any abnormalities.

\section{Investigations:}

- Iron Status: The mother and her baby's blood profiles included hemoglobin $(\mathrm{Hb})$ and serum ferritin (SF). Blood samples were taken from mothers and their babies as follows: For Ferritin: One milliliter of venous blood was taken on serum separator tube (SST) and allowed to clot then sent directly to the laboratory to obtain result for analysis. - For CBC: One milliliter of venous blood was taken on ethylene diamine tetra acetic acid (EDTA) tube and assayed immediately for complete blood picture by automated way. The obtained parameters were: (Hemoglobin concentration, hematocrit value, RBCs count, platelets count, and total leucocytic count).

- Otoscopic examination by Immittancemetry: (Immittacemetry of Interacoustics AT235h) That including: - High Frequency Tympanometry: (probe tone $1 \mathrm{KHz}$ ): Assessed after 2 weeks of birth and was done at varying pressure ranging from -400 to $+200 \mathrm{mmH}_{2} \mathrm{O}$ - Acoustic reflex: Ipsilateral acoustic reflex was done for each ear by using pure tones of 500, 1000, 2000 and $4000 \mathrm{~Hz}$. Doppler echocardiography: for diagnosis of type of cardiac disease and assessment of cardiac function.

- Otoacoustic Emissions test: This was done for all babies with normal tympanometric pressure and normal acoustic reflex. It was performed by observing the changes of transient evoked otoacoustic emissions (TEOAE) and distortion product otoacoustic emissions (DPOAE) and recording the responses as (pass - partially pass - fail) by using (Otoacoustic emissions of intelligent hearing system (IHS) 


\section{Statistical analysis}

Statistical analysis was done using IBM SPSS version 23. Data were expressed as mean $\pm \mathrm{SD}$, range, median, frequency and percentage and were analyzed using the independent student " $t$ " test and chi-square

\section{RESULTS}

Laboratory assessments of the measured parameters are presented in the following tables and figures:

Table (1) Comparison between the two studied groups according to their sex

\begin{tabular}{|c|c|c|c|c|c|c|}
\hline \multirow{2}{*}{ Babies' sex } & \multicolumn{2}{|c|}{ Control $(\mathbf{n}=\mathbf{3 0})$} & \multicolumn{2}{|c|}{ IDA cases $(\mathbf{n}=\mathbf{3 0})$} & \multirow{2}{*}{ Test of sig. } & \multirow{2}{*}{ P } \\
\cline { 2 - 5 } & No. & $\mathbf{\%}$ & No. & \% & & 1.000 \\
\hline Male & 14 & 46.7 & 14 & 46.7 & $\square \square \square \square \square$ & \\
\hline Female & 16 & 53.3 & 16 & 53.3 & & \\
\hline
\end{tabular}

- Table (1) showed that there was no statistically significant difference as regards the babies sex in the control and study groups $(\mathrm{p}>0.05)$.

Table (2) Comparison between the two studied groups as regards mothers' $\mathrm{Hb}$ and babies'ferritin

\begin{tabular}{|c|c|c|c|c|}
\hline Babies' Ferritin & Control $(\mathbf{n}=\mathbf{3 0})$ & IDA cases $(\mathbf{n}=\mathbf{3 0})$ & Test of sig. & P \\
\hline Min. - Max. & $88.0-189.0$ & $22.10-194.60$ & $\mathrm{t}=$ & $0.012^{*}$ \\
\hline Mean \pm SD. & $124.25 \pm 28.54$ & $97.37 \pm 48.55$ & & \\
\hline Median & 123.0 & 93.0 & & \\
\hline Mothers' HB & & & \multirow{2}{*}{$14^{*}$} & $<0.001^{*}$ \\
\hline Min. - Max. & $11.0-12.40$ & $7.30-9.80$ & $19.200^{*}$ & \\
\hline Mean \pm SD. & $11.72 \pm 0.41$ & $8.87 \pm 0.70$ & & \\
\hline Median & 11.80 & 9.15 & & \\
\hline
\end{tabular}

- Table (2) showed that There was significant difference as regards babies'serum ferritin level and their mothers'hemoglobin level $(\mathrm{p}<0.05)$.

Table (3): Correlation between Babies' ferritin and mothers' Hb in each group

\begin{tabular}{|l|c|c|c|c|}
\hline \multirow{2}{*}{ Baby ferritin vs. mother HB } & \multicolumn{2}{|c|}{ Control } & \multicolumn{2}{c|}{ Cases } \\
\cline { 2 - 5 } & $\mathbf{r}$ & $\mathbf{p}$ & $\mathbf{r}$ & $\mathbf{P}$ \\
\cline { 2 - 5 } & 0.234 & 0.213 & 0.199 & 0.291 \\
\hline
\end{tabular}

- Table (3) showed that there was no statistically significant correlation between baby ferritin and mother hemoglobin $(\mathrm{P}>0.05)$.

Table (4): Comparison between the two studied groups according to screening of TEOAE and their ferritin level

\begin{tabular}{|l|c|c|c|c|c|c|}
\hline Screening of TEOAE & \multicolumn{2}{|c|}{ Control $(\mathbf{n}=\mathbf{3 0})$} & \multirow{2}{*}{$\begin{array}{c}\text { Mean Ferritin } \\
\text { level }\end{array}$} & \multicolumn{2}{c|}{ IDA cases (n= 23) } & \multirow{2}{*}{$\begin{array}{c}\text { Mean Ferritin } \\
\text { level }\end{array}$} \\
\hline Right & No. & $\boldsymbol{\%}$ & & No. & \% & 102.89 \\
\hline Pass & 30 & 100.0 & 124.25 & 16 & 69.6 & 135.58 \\
\hline Partially Pass & 0 & 0.0 & 0.0 & 5 & 21.7 & 50.05 \\
\hline Fail & 0 & 0.0 & 0.0 & 2 & 8.7 & \\
\hline Left & & & & & & 94.95 \\
\hline Pass & 30 & 100.0 & 124.25 & 20 & 87.0 & 91.90 \\
\hline Partially Pass & 0 & 0.0 & 0.0 & 3 & 13.0 & \\
\hline
\end{tabular}

- Table (4) showed that the comparing the pass - fail results for TEOAEs and DPOAEs between control and study groups in right and left ears. 
Table (5): Comparison between the two studied groups according to screening of DPOAE and their ferritin level

\begin{tabular}{|l|c|c|c|c|c|c|}
\hline Screening of DPOAE & \multicolumn{2}{|c|}{ Control (n =30) } & \multirow{2}{*}{$\begin{array}{c}\text { Mean Ferritin } \\
\text { level }\end{array}$} & IDA cases (n= 23) & \multirow{2}{*}{$\begin{array}{c}\text { Mean Ferritin } \\
\text { level }\end{array}$} \\
\hline Right & No. & $\mathbf{\%}$ & & No. & \% & 74.71 \\
\hline Pass & 30 & 100.0 & 124.25 & 7 & 30.4 & 118.83 \\
\hline Partially Pass & 0 & 0.0 & 0.0 & 16 & 69.6 & \\
\hline Left & & & & & & 67.36 \\
\hline Pass & 30 & 100.0 & 124.25 & 5 & 21.7 & 102.11 \\
\hline Partially Pass & 0 & 0.0 & 0.0 & 18 & 78.3 & \\
\hline
\end{tabular}

- Table (5) showed that in DPOAEs all healthy babies (control group) passed the test while for IDA cases $30.4 \%$ passed the test as regards Rt ear and $21.7 \%$ as regards Lt ear and no cases failed.

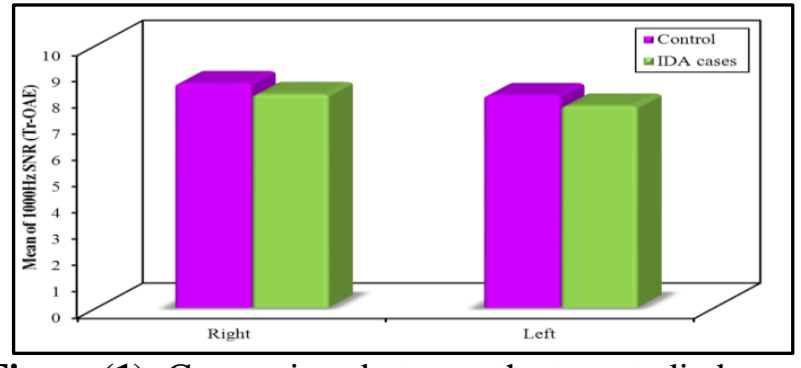

Figure (1): Comparison between the two studied groups according to $1000 \mathrm{~Hz}$ SNR (TEOAE)

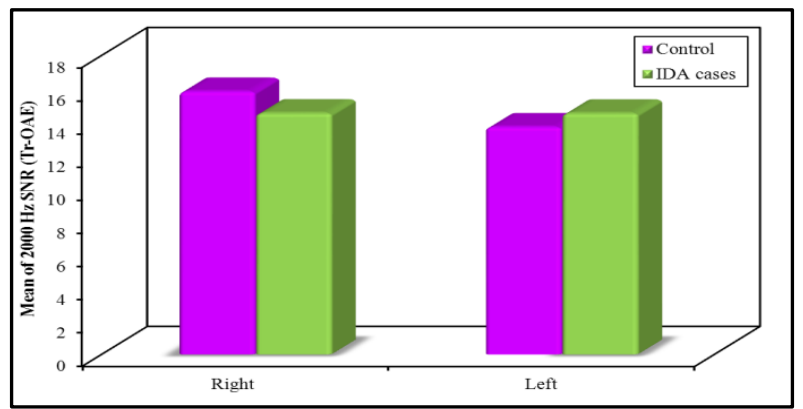

Figure (3): Comparison between the two studied groups according to $2000 \mathrm{~Hz}$ SNR (TEOAE)

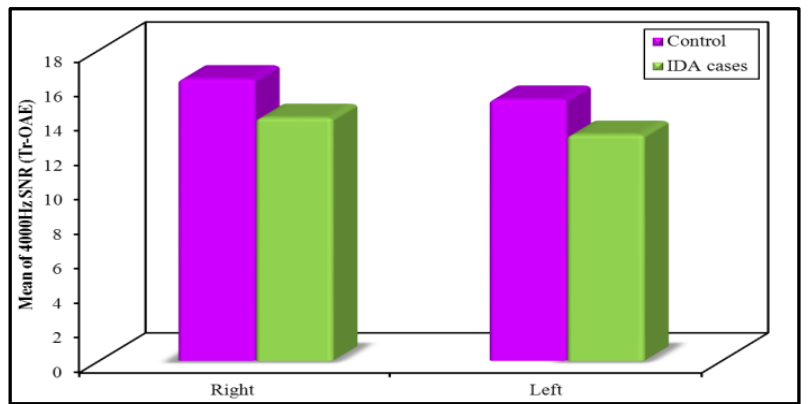

Figure (5): Comparison between the two studied groups according to $4000 \mathrm{~Hz}$ SNR (TEOAE)

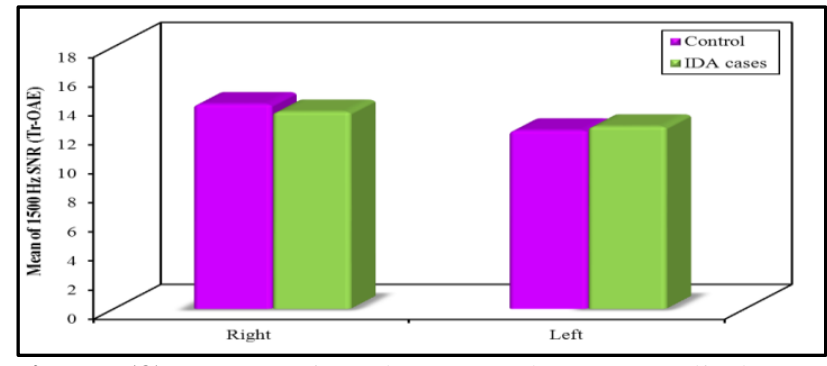

Figure (2): Comparison between the two studied groups according to $1500 \mathrm{~Hz}$ SNR (TEOAE)

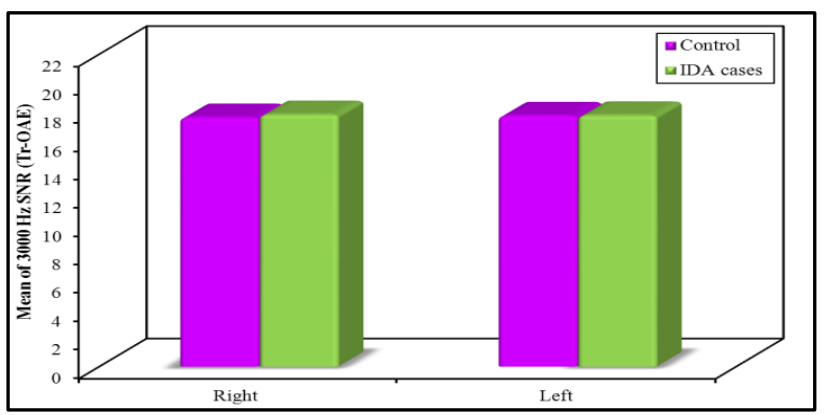

Figure (4): Comparison between the two studied groups according to $3000 \mathrm{~Hz}$ SNR (TEOAE)

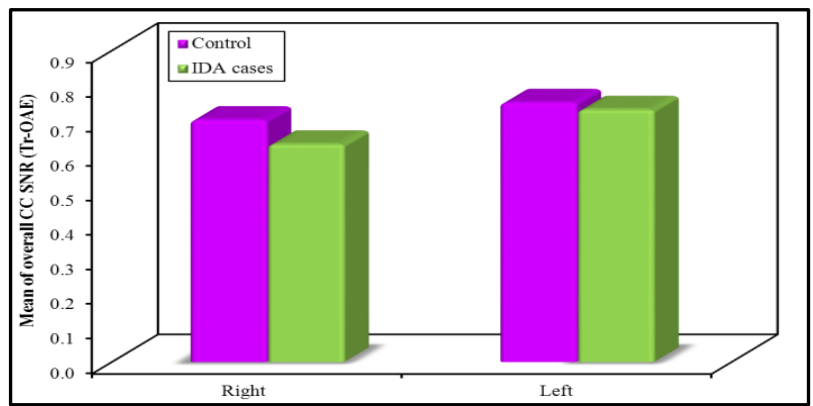

Figure (6): Comparison between the two studied groups according to overall cc (TEOAE)

- Figures 1-6 showed the comparison between the two groups as regards the TEOAEs; overall amplitude and signal to noise ratio (SNR) at different frequencies showed statistically significant difference at frequency of $4000 \mathrm{~Hz}$ in both right and left ears $(\mathrm{p}<0.05)$. The comparison between right and left ears as regards signal to noise ratio (SNR) and overall amplitude showed that there was statistically significant difference as regards the frequency at $2000 \mathrm{~Hz}$ in control group and $4000 \mathrm{~Hz}$ in study group $(\mathrm{p}<0.05)$ and there was tendency for right ear to give higher amplitude than left ear. 


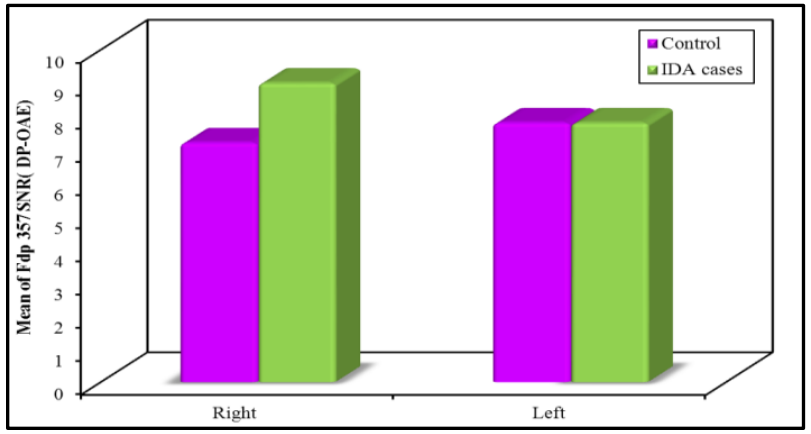

Figure (7): Comparison between the two studied groups according to Fdp 357 SNR (DPOAE)

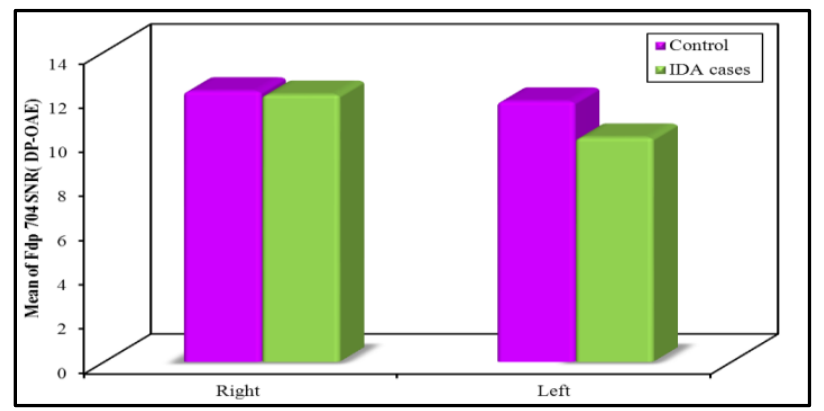

Figure (9): Comparison between the two studied groups according to Fdp 704 SNR (DPOAE)

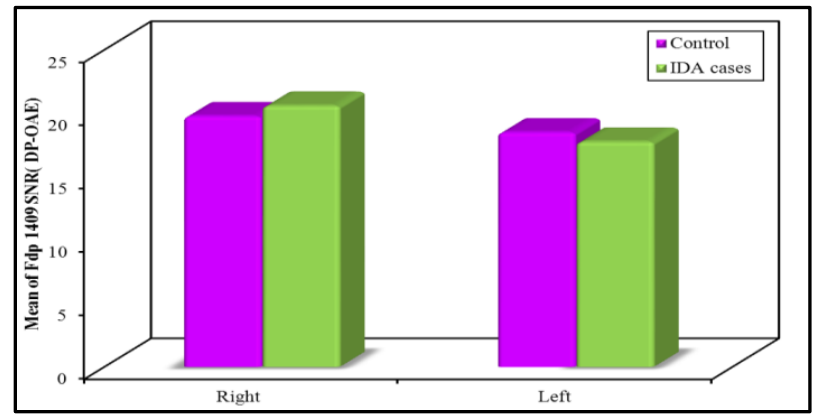

Figure (11): Comparison between the two studied groups according to Fdp 1409 SNR (DPOAE)

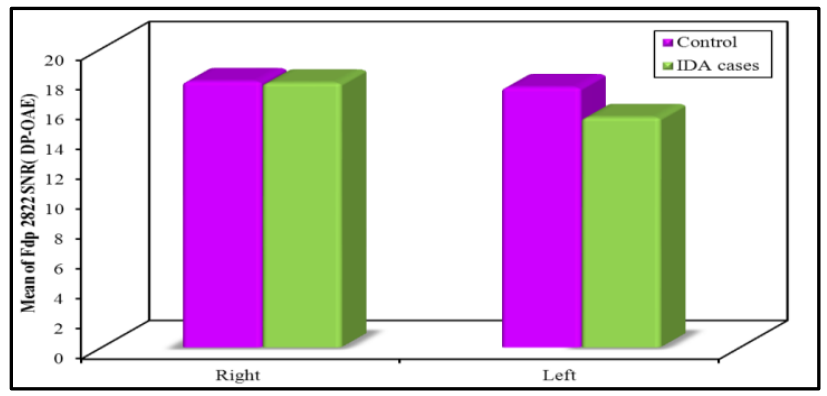

Figure (13): Comparison between the two studied groups according to Fdp 2822 SNR (DPOAE)

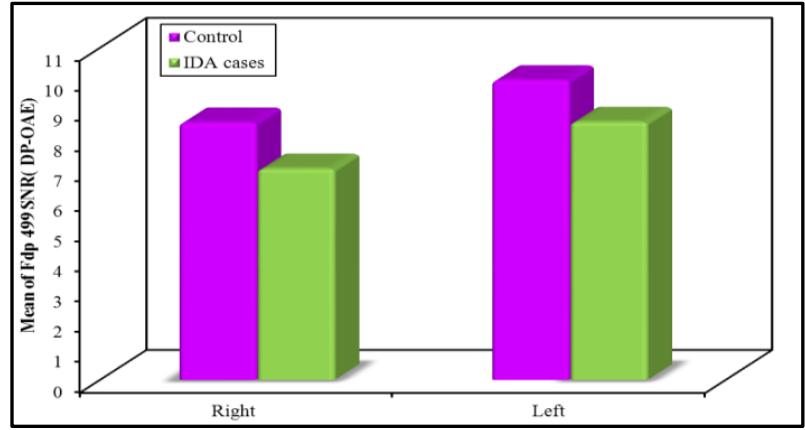

Figure (8): Comparison between the two studied groups according to Fdp 499 SNR (DPOAE)

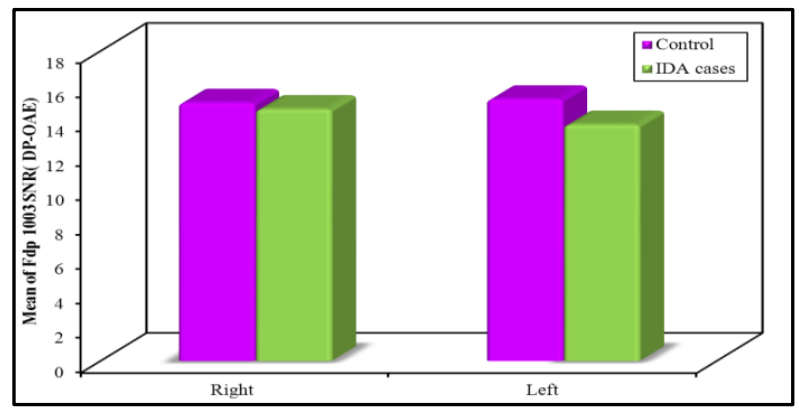

Figure (10): Comparison between the two studied groups according to Fdp 1003 SNR (DPOAE)

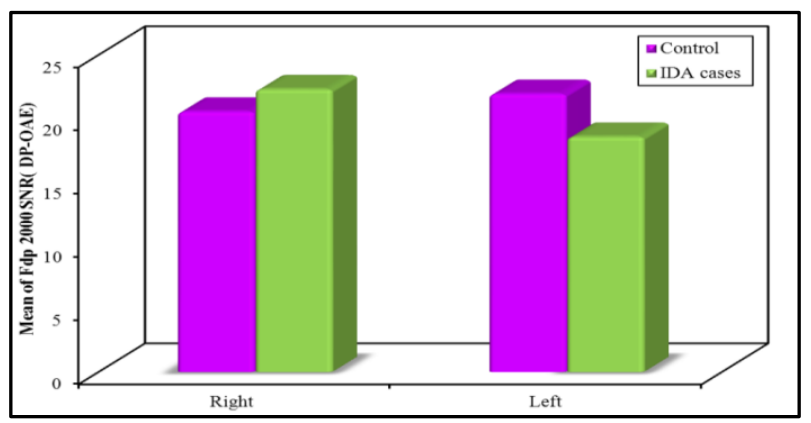

Figure (12): Comparison between the two studied groups according to Fdp 2000 SNR (DPOAE)

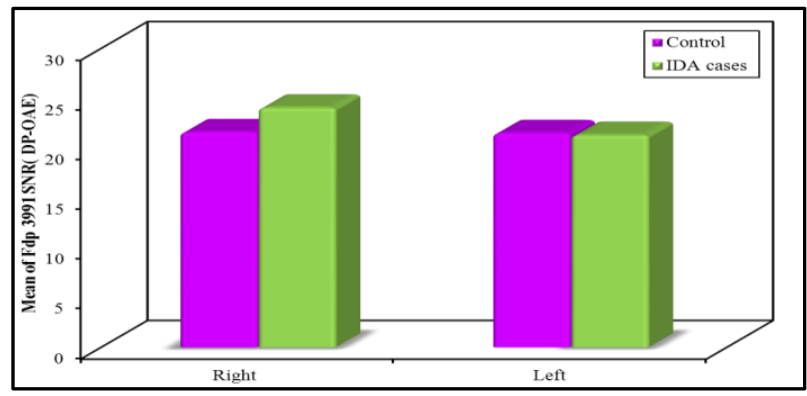

Figure (14): Comparison between the two studied groups according to Fdp 3991 SNR (DPOAE) 


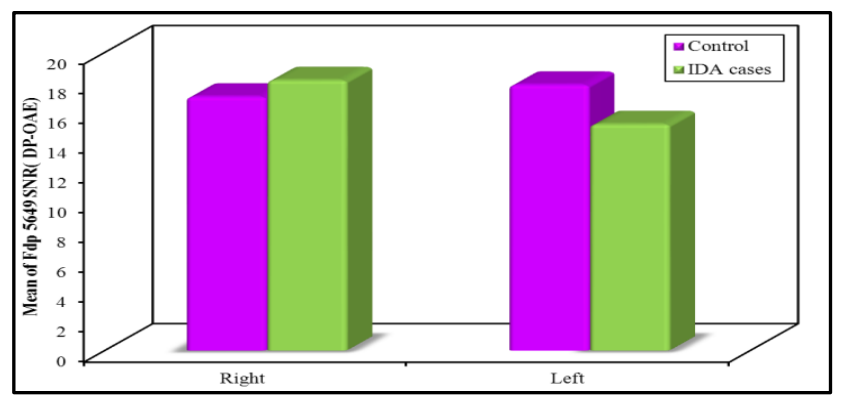

Figure (15): Comparison between the two studied groups according to Fdp 5649 SNR (DPOAE)

- Figures 7-15 showed the comparison between the two groups as regards signal to noise ratio (SNR) at different frequencies. There was no significant difference in frequencies between the two groups ( $\mathrm{p}>0.05)$.

- The comparison between right and left ears showed that there was statistically significant difference as regards signal to noise ratio at frequencies 1409 and $2822 \mathrm{~Hz}$ of study group $(\mathrm{p}<0.05)$.

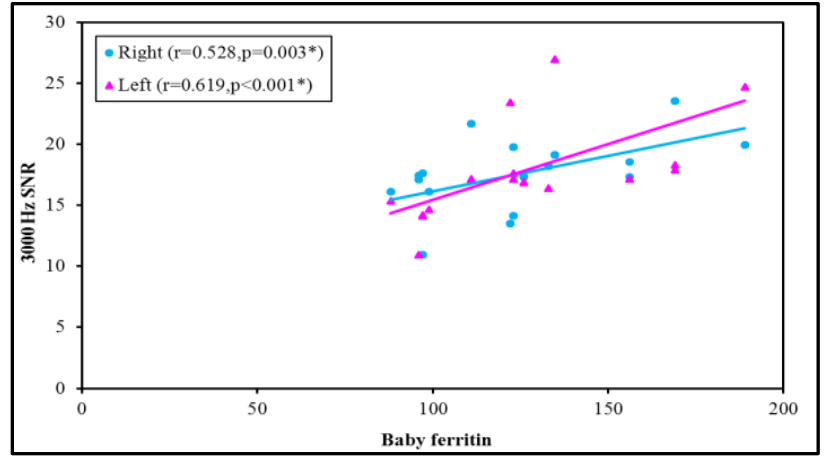

Figure (16): Correlation between babies' ferritin and $3000 \mathrm{~Hz}$ SNR in control group $(\mathrm{n}=30)$

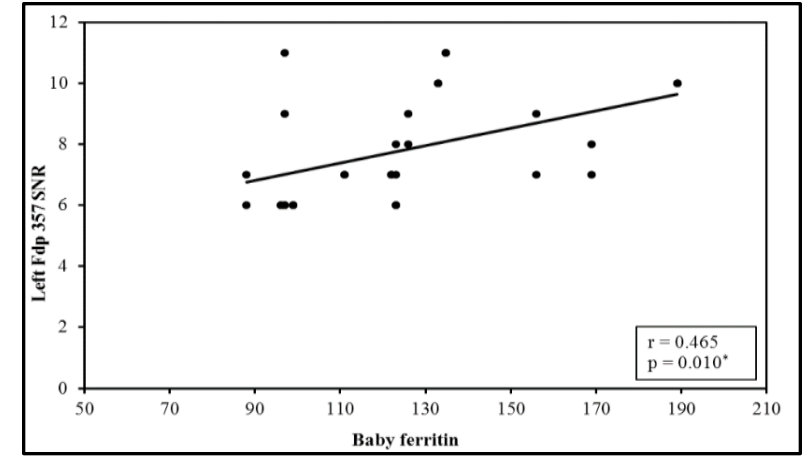

Figure (17): Correlation between babies' ferritin and left Fdp 357 SNR in control group $(n=30)$

- As regards control group; figures 16-17 showed that there was statistically significant correlation between babies' ferritin and SNR in TOAEs at frequency $3000 \mathrm{~Hz}$ in both right and left ears and with SNR in DPOAEs at frequency $357 \mathrm{~Hz}$ in left ear, and there was no statistically significant correlation between mothers' $\mathrm{Hb}$ and SNR in both TEOAEs and DPOAEs.

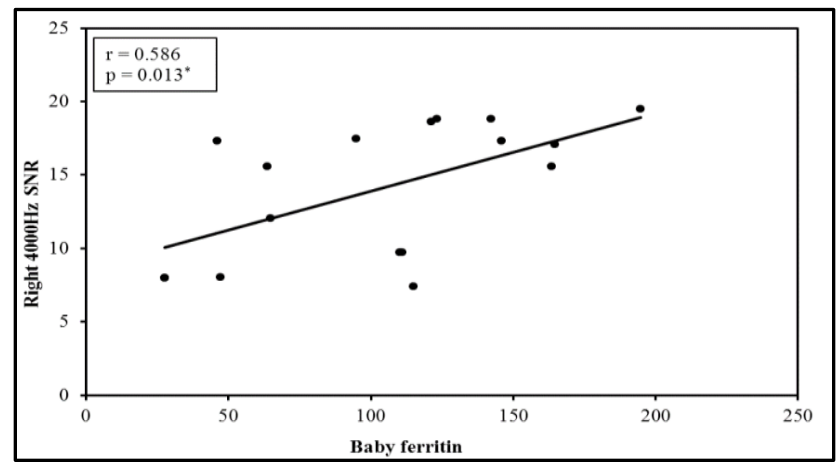

Figure (18): Correlation between babies' ferritin and right $4000 \mathrm{~Hz}$ SNR (TEOAE) in IDA group $(\mathrm{n}=30)$

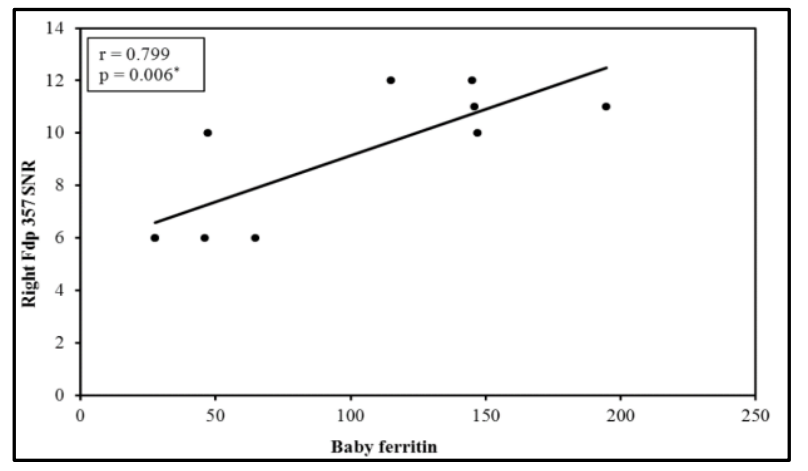

Figure (19): Correlation between babies' ferritin and right Fdp 357 SNR in IDA group $(n=30)$

- As regards study group; figures 18-19 showed that there was statistically significant correlation between babies' ferritin and SNR in TEOAEs at frequencies $4000 \mathrm{~Hz}$ in right ear and with SNR in DPOAEs at frequencies $357 \mathrm{~Hz}$ in right ear, and there was no statistically significant correlation between mothers' Hb and SNR in both TEOAEs and DPOAEs.

\section{DISCUSSION}

Iron deficiency may lead to functional and structural changes in the cochlea since some of the cochlear enzymes contain iron. However, cochlear function analysis by otoacoustic emission in patients with iron deficiency was reported in a few studies before (3).

The results of the current study revealed that there was significant difference between control and study 
groups as regards babies' serum ferritin level and mothers' hemoglobin level $(\mathrm{p}<0.05)$ but no correlation was found between them. This result agreed with the result of a study done by Betelihem et $\boldsymbol{a l}$. $^{(8)}$ who included 89 mothers and their respective newborns and performed complete blood count and serum ferritin. Maternal median hemoglobin and serum ferritin levels were $12.2 \mathrm{~g} / \mathrm{dL}$ and $47.0 \mathrm{ng} / \mathrm{mL}$, respectively. The median hemoglobin and serum ferritin levels for the newborns were $16.2 \mathrm{~g} / \mathrm{dL}$ and $187.6 \mathrm{ng} / \mathrm{mL}$, respectively. Newborns of IDA mothers had significantly lower levels of serum ferritin and hemoglobin concentration. They concluded that maternal IDA may have an effect on the iron stores of newborns and this agreed with the current study results that babies of IDA mothers had lower ferritin levels than control group.

A survey carried out in a rural area of southeastern China ${ }^{(9)}$, where most of the women had iron deficiency anemia and most newborns did not have anemia and showed elevated ferritin levels. One possible explanation is the fact that iron stores in the fetus are not adversely affected by mild to moderate maternal anemia, reinforcing the theory that iron is transported across the placenta regardless of its level in the maternal blood $^{(\mathbf{1 0})}$. These results are in agreement with the study of Chopard et al. (11), who reported that mild or moderate maternal iron deficiency anemia does not cause low iron stores (ferritin) in the fetus, but can progress to preterm labor and newborns with low birth weight.

Gaspar et al. (12) studied 157 pregnant women during their third trimester of pregnancy and their newborns at birth, analyzing the mother's and the umbilical cord's blood for hemoglobin and serum ferritin. They observed that the blood levels in the umbilical cord did not decrease until the ferritin value of the mother was $<12$ micrograms/l.

The present study compared the pass - fail results of TEOAEs and DPOAE between control and study groups.

TEOAE can be detected essentially in all normally hearing ears with hearing threshold better than $30 \mathrm{~dB}$ HL. They are frequently used for hearing screening in newborns, patients who have been using ototoxic drugs and noise exposed workers. DPOAE are generated by two stimulus tones of moderate level (55$75 \mathrm{~dB}$ SPL) separated in frequency, presented to the ear measured over a frequency range from 0.5 to $8 \mathrm{kHz}$ so they are absent with hearing threshold less than $55 \mathrm{~dB}$ HL ${ }^{(13-15)}$.

Santos et al. ${ }^{(16)}$ concluded that anemic children differed statistically from non-anemic children in terms of alterations in the acoustic reflex and language development and also presented a higher prevalence of alterations in the hearing evaluation. The research group was composed of 19 anemic children and the control group of 38 healthy children, selected through randomized paired sampling. The children's hearing was evaluated through otoacoustic emissions and tympanometry. They observed a statistically significant clinical finding $(\mathrm{p}=0.08)$, showing that the anemic children fail more often (52\%) in the first hearing assessment than the non-anemic (28\%). Other studies demonstrated that these retest indexes are higher in hearing screening programs, not considering nutritional conditions. In this manner, it was observed that children with anemia have more vulnerability to peripheral hearing impairment. ${ }^{(17)}$

The correlation between the sensori-neural hearing loss and iron deficiency was subject of various studies. Sun et al. (18) compared the ferritin concentration between healthy subjects with normal hearing and patients with different kinds of sensorineural hearing loss. They found lower ferritin levels in patients with hearing loss.

There are reports in the literature indicating that iron deficiency may cause hearing impairment, which actually presents some conflicting results. Tobiášková and Petržílková (19) analyzed and compared physiological and risk newborns examined with otoacoustic emission and found in the personal medical history of mothers of children with hearing loss, the most frequently repeated disease was diabetes mellitus (44.44\%), whose relationship with hearing impairment was addressed in several studies in the USA. Other diseases that cause hearing loss is for example hypothyroidism, which occurred in $(33.33 \%)$. Both gestational anemia and preeclampsia was $(11.11 \%)$ for each, so they concluded that the incidence of hearing impairments occurs in risk newborns as well as physiological.

In the present study, transient evoked otoacoustic emissions revealed significant difference between the studied groups at frequency of $4000 \mathrm{~Hz}$ in both right and left ears. We also compared between right and left ears as regards signal to noise ratio (SNR) and overall amplitude. There was significant difference at the frequency of $2000 \mathrm{~Hz}$ in control group and at $4000 \mathrm{~Hz}$ in study group. We also revealed that there was no significant difference between mean SNR of males and females but we detected that mean SNR of females mostly higher than males.

Ravi and Naresh (20) carried out a study on normal hearing infants and children and used parameters of TEOAE. They concluded that there was no significant difference between mean responses of right 
and left ears and they detected that females in neonatal group has higher amplitude and SNR than males.

In the present study, distortion product otoacoustic emissions revealed no significant difference as regard SNR at different frequencies between the two studied groups $(\mathrm{p}>0.05)$. We also compared between right and left ears as regard signal to noise ratio (SNR). There was significant difference in study group with right ears showing a higher mean SNR than left ears and there was no significant difference in control group. We also revealed that in control group; there was significant difference where mean SNR of females mostly higher than males. In study group; there was significant difference between mean SNR of males and females at frequencies $3991 \mathrm{~Hz}$ at both right and left ears and 704 $\mathrm{Hz}$ at right ear only with mean SNR of females higher than males.

Keogh et al. ${ }^{(21)}$ carried out a study to examine the effects of ear asymmetry and gender on distortionproduct otoacoustic emissions (DPOAEs) obtained from children (528 boys and 475 girls), with a mean age of 6.2 years $(\mathrm{SD}=0.4$, range $=5.2-7.9$ years $)$, were tested in a quiet room at their schools using the GSI- 60 DPOAE system. The results revealed a small but significant difference in SNR between ears, with right ears showing a higher mean SNR than left ears at 1.9, $3.0,3.8$, and $6.0 \mathrm{kHz}$. At these frequencies, the difference in mean SNR between ears was less than 1 dB. A significant gender effect was also found. Girls exhibited a higher SNR than boys at 3.8, 4.8, and 6.0 $\mathrm{kHz}$. The difference in mean SNR, as a result of the gender effect, was about 1 to $2 \mathrm{~dB}$ at these frequencies.

Pinto and Lewis (22) carried out a study to describe the DPOAE records of infants from birth to two months, considering the response level, noise level and the signal to noise ratio in all frequencies; the analysis of response level according to the variables of gender, tympanometry pressure peak, state of infant during the test and distribution of the response percentile level. The study was done on 138 infants who were evaluated. All of them presented no risk indicators for hearing loss and passed the hearing screening test, 70 male and 68 female were evaluated, with ages between 6 to 65 days. They concluded that there was no statistically significant difference between genders and between ears for the response level. The tympanometry pressure peak indicated no influence on records of the response level. As regards to the infant's state during the test (light sleep, deep sleep and alert without body activity) did not influence the recording of the DPOAE response level. The background noise influenced the identification and the analysis of DPOAE at frequencies (f2) below 2002 Hz. Finally, we carried out the correlation between babies' ferritin and mothers' Hb with SNR in TEOAE
\& DPOAE and found that there was no statistically significant correlation between mothers' $\mathrm{Hb}$ and SNR in TEOAEs and in DPOAEs in both control and study groups. As regards babies' ferritin there was statistically significant correlation with SNR in TEOAEs at high frequencies 3000 and $4000 \mathrm{~Hz}$ in control and study group in both right and left ears and with SNR in DPOAEs at low frequency $357 \mathrm{~Hz}$.

Sun et al. ${ }^{(18)}$ reviewed the outcome of 426 patients with idiopathic sudden hearing loss in 13 years and found low hemoglobin $(42.4 \%)$ and serum ferritin $(22.3 \%)$ levels. They observed better recovery with the iron therapy and they postulated that reversible change in enzymatic activity might be important biochemical basis for spontaneous recovery occurring in some cases of sudden sensorineural hearing loss. The severity of anemia does not seem to be associated with the severity of hearing loss. However, the longer the duration of iron deficiency, the higher the incidence of hearing loss.

In the present study, the effect of iron deficiency on the function of the auditory system was investigated by cochlear emissions. Experimental studies indicated that iron deficiency anemia may affect the auditory function to a certain extend. The purpose of the current study was to establish the presence or absence of hearing impairment in babies of iron deficiency anemia mothers by means of otoacoustic emission. The results of the present study did not support a causal relationship between the iron deficiency anemia and the auditory dysfunction on the basis of DPOAE.

Ozturan et al. ${ }^{(23)}$ carried out an experimental study and auditory function was tested in iron-deficient and normal male Wistar albino rats using distortion product otoacoustic emissions and auditory brainstem response audiometry. Hemoglobin, hematocrit, serum iron and albumin levels were monitored to verify iron deficiency. Although dramatic differences in weight gain and blood test parameters were noted, no significant change in auditory function due to iron deficiency was detected.

Fei et al. ${ }^{(24)}$ carried out a study to evaluate whether mild maternal IDA in guinea pigs could interrupt inner hair cell (IHC) ribbon synapse density and outer hair cell motility of the offspring. Hearing function was evaluated by auditory brainstem response $(\mathrm{ABR})$ and distortion product otoacoustic emission (DPOAE) measurements. Then, the cochleae were harvested for assessment of the number of IHC ribbon synapses by immunofluorescence, the morphology of cochlear hair cells, and spiral ganglion cells (SGCs) by scanning electron microscope and hematoxylin-eosin staining. The results showed that mild maternal IDA in guinea pigs induced elevated ABR threshold shifts, declined DPOAE level shifts, reduced the number of 
ribbon synapses, and impaired the morphology of cochlear hair cells and SGCs in offspring. These data indicate that mild maternal IDA in guinea pigs induced hearing impairment in offspring, and this deficit may be attributed to the reduction of ribbon synapse density.

Rakesh et al. ${ }^{(25)}$ carried out a study of 400 cases to investigate the potential role of transient-evoked otoacoustic emissions (TEOAEs) beyond screening for hearing impairment in different middle/inner ear disorders in 3-65 years age group. They concluded that TEOAEs is a reliable, simple and cost effective screening technique for hearing disorders and they recommended $\mathrm{OAE}$ as a suitable primary neonatal hearing screening all over the country.

In conclusion; neonates with maternal IDA were affected in their hearing abilities. The peripheral auditory function, as tested by TEOAEs test, was affected in high frequency region of the cochlea in low ferritin babies while DPOAEs test was less sensitive in these patients for assessment of their cochlear function; this can be explained by the possibility of mild hearing loss which can be detected by TEOAE (present with hearing threshold better than $30 \mathrm{~dB}$ HL) while not by DPOAE (absent with hearing threshold worse than 55 $\mathrm{dB}$ HL). Therefore, TEOAE is the most sensitive test in detection of early hearing affection in IDA patients.

\section{EFERENCES}

1. Baig-Ansari N, Badruddin S, Karmaliani $R$ et al. (2008): Anemia prevalence and risk factors in pregnant women in an urban area of Pakistan. Food Nutr Bull. , 29(2): 132-139.

2. World Health Organization (2016): Centers for Disease Control and Prevention, WHO recommendation on daily oral iron and folic acid supplementation. Geneva, Switzerland. WHO/UNICEF/UNU. P. 123.

3. Shao J, Lou J, Rao $\mathbf{R}$ et al. (2012): Maternal serum ferritin concentration is positively associated with newborn iron stores in women with low ferritin status in late pregnancy. J Nutrition, 142(11): 2004-9.

4. Janssen T, Niedermeyer $H$ and Arnold $W$ (2006): Diagnostics of the cochlear amplifier by means of distortion product otoacoustic emissions. ORL J Otorhinolaryngol Relat Spec., 68(6): 334-339.

5. Breymann C (2015): Iron Deficiency Anemia in Pregnancy. Semin Hematol., 52(4): 339-47.

6. World Health Organization (WHO) (2001). Iron Deficiency Anemia Assessment, Prevention and Control. A guide for programmer managers. Geneva: WHO/UNICEF/UNU. P. 114.

7. Ballard J, Khoury J, Wedig K et al. (1991): New Ballard Score expanded to include extremely premature infants. J Pediatr. , 119(3): 417-23.

8. Betelihem T, Asaye B, Paulos $N$ et al. (2015): Effect of Maternal Iron Deficiency Anemia on the Iron Store of Newborns in Ethiopia. Anemia, 6: 1-5.
9. Dani C, Rossetto S, Castro S et al. (2008): Prevalence of anemia and nutritional deficiencies, through different laboratory parameters, in pregnant women attended at two public health services in Rio Grande do Sul. Rev Bras Anal Clin., 40: 171-5.

10. Reihaneh H, Norimah A, Poh B et al. (2010): Haemoglobin and serum ferritin levels in newborn babies born to anaemic Iranian women: A cross-sectional study in an Iranian hospital. Pakistan J Nutr.,9(6): 562-566.

11. Chopard T, Maurício $M$ and Paula B (2010): Iron deficiency in the fetus and newborn. Revista Brasileira de Hematologia e Hemoterapia, 32: 22-66.

12. Gaspar M, Ortega $R$ and Moreiras $O$ (1993): Relationship between iron status in pregnant women and their newborn babies. Investigation in a Spanish population. Acta Obstet Gynecol Scand.,72(7): 534-7.

13. Lonsbury-Martin B and Martin G (1990): The clinical utility of distortion product otoacoustic emissions. Ear Hear, 11: 144-154.

14. Martin G, Ohlms L, Franklin D et al. (1990): Distortion product emissions in humans III. Influence of sensorineural hearing loss. Ann Otol Rhinol Laryngol., 99: 30-42.

15. Probst R, Lonsbury-Martin $B$ and Martin G (1991): A review of otoacoustic emissions. J Acoust Soc A.,, 89: 2027-67.

16. Santos J, Lemos $\mathbf{S}$, Rates $\mathbf{S}$ et al. (2008): Hearing abilities and language development in anemic children of a public daycare center. Pró-Fono R. Atual. Cient., 20 (4): 150-210.

17.Holm J, Sørensen $C$ and Tos M (1981): Repetitive tympanometric screenings in 4-year-old children. Seasonal influence on secretory otitis and tubal dysfunction. ORL J Otorhinolaryngol Relat Spec., 43(3): 164-74.

18. Sun A, Wang Z, Xiao S et al. (1992): Idiopathic sudden hearing loss and disturbance of iron metabolism. A clinical survey of 426 cases. ORL.,54: 66-70.

19. Tobiášková $L$ and Petržílková H (2015): Otoacoustic emissions screening in newborn babies. Peer-Reviewed Journal for NonMedical Health Professions, 8(2): 1803-4330.

20. Ravi K and Naresh K (2006): Transient Evoked Otoacaustic Emissions. Indian J Pediatr., 73(4):283-286.

21.Keogh T, Kei J, Carlie J et al. (2001): Distortion-product otoacoustic emissions in schoolchildren: Effects of ear asymmetry, handedness, and gender. Journal of the American Academy of Audiology, 12(10): 506-13.

22. Pinto VS and Lewis DR (2007): Distortion product otoacoustic emissions in infants from birth to two months. Pró-Fono R. Atual. Cient., 19 (2): 133-140.

23. Ozturan O, Henley C, Littman T et al. (1997): Iron deficiency anemia and hearing. ORL J Otorhinolaryngol Relat Spectrosc, 59(2): 73-8.

24. Fei Y, Shuai H, Bo Y et al. (2016): Mild Maternal Iron Deficiency Anemia Induces Hearing Impairment Associated with Reduction of Ribbon Synapse Density and Dysregulation of VGLUT3, Myosin VIIa, and Prestin Expression in Young Guinea Pigs. Neurotox Res., 29: 594- 604.

25. Rakesh S, Deepali M, Dinesh B et al. (2013): Role of Transient Evoked Otoacoustic Emission Beyond Screening of Hearing Impairment: A Study of 400 Cases. Indian J Otolaryngol Head Neck Surg., 65(2): 134-139. 